\title{
Adherence to international recommendations of physiotherapy in Chilean adult intensive care units
}

\begin{abstract}
International recommendations propose the presence of physiotherapists with specialized training in critical patients, available 24 hours a day and 7 days a week (24/7-PT) in the Intensive Care Unit (ICU), with a standardized physiotherapist/patient ratio of 1 per 5 beds in high complexity units. It is unknown if the organizational characteristics of the ICUs are related to the adherence with these recommendations.
\end{abstract}

Objective: To determine the relationship between the organizational characteristics and the adherence of international recommendations of physiotherapy care, in adult ICUs at a national level.

Methods: Secondary database analysis, with information from $86 \%$ of adult Chilean ICUs. The organizational characteristics (origin of the ICU, number of beds, level of complexity and technical-administrative dependency of the physiotherapist) were related to 24/7-PT availability, physiotherapist/patient ratio of 1 per 5 beds and presence of physiotherapy specialists.

Results: Out of 64 adult ICUs, a 70\% (n=45) have 24/7-PT availability. Physiotherapist/ patient ratio was observed during weekdays in half of the private centers during day shifts. Presence of physiotherapy specialists was detected in $31 \%$ of the private sector. Significant differences were found between the number of beds and the probability to accomplish 24/7-PT availability and physiotherapist/patient ratio $(\mathrm{p}<0.001$ and $\mathrm{p}=0.34$ respectively). The probability that a unit complies $24 / 7-\mathrm{PT}$ availability differs significantly depending on the technical-administrative dependency $(p<0.001)$. No significant relationship was found between the level of complexity and the adherence to the international recommendations. A greater adherence to $24 / 7$-PT availability in public ICUs (OR 5.21, 95\%CI 1.37-19.81) and when physiotherapist-dependence became the Critical Patients Unit (OR 6.59, 95\%CI 1.41-30.80) was evidenced.

Conclusions: The origin of the ICU and the technical-administrative dependence seem to be relevant for the compliance of 24/7-PT availability. Future studies should be aimed at identifying the causes of this relationship, in order to optimize the human resources in the ICU.

Keywords: critical care [mesh], physical therapy specialty [mesh], physical therapy department, hospital [mesh], organization and administration [mesh], recommendations, workload [mesh]

Abbreviations: CI, confidence interval; ESICM, european society of intensive care medicine; HDU, high dependency unit; ICU, intensive care unit; OR, odds ratio; SOCHIMI, chilean society of intensive medicine; 24/7-PT, 24 hours a day and 7 days a week physiotherapist.

\section{Introduction}

Evidence suggests that early rehabilitation in the Intensive Care Units (ICU) can contribute to decrease the number of days of respiratory support, the length of stay in hospital, the incidence of respiratory infections and the mortality rate. ${ }^{1-4}$

In 2011, the European Society of Intensive Care Medicine (ESICM) issue a guideline with the basic requirements for ICUs. It recommended that level III units (high complexity) should be staffed with one specialist physiotherapist every five beds, twenty-four hours a day and seven days a week (24/7). ${ }^{5}$ In Chile, the Chilean Society of Intensive Medicine (SOCHIMI by its initials in Spanish) recommends having a post-graduate training physiotherapist available 24 hours a day in ICUs without referring to a safe staffing level. ${ }^{6}$

Current global trend invites standardization of care resources to favor better functioning of the units, improve the quality of care and

\author{
Volume 4 Issue 2 - 2019
}

\author{
Nadine Aranis,' Jorge Molina, ${ }^{2}$ Jaime Leppe, ${ }^{2}$ \\ Ana Cristina Castro-Ávila, ${ }^{2,3}$ Carolina Fu, ${ }^{4}$ \\ Catalina Merino-Osorio ${ }^{2}$ \\ 'Physical Medicine and Rehabilitation Service, Clínica Alemana, \\ Chile \\ ${ }^{2}$ Facultad de Medicina Clínica Alemana Universidad del \\ Desarrollo, Chile \\ ${ }^{3}$ Department of Health Sciences, University of York, United \\ Kingdom \\ ${ }^{4}$ Departament of Physical Therapy, Speech and Occupational \\ Therapy, Faculty of Medicine -Universidade São Paulo, Brasil
}

Correspondence: Nadine Aranis. Physical Medicine and Rehabilitation Service, Clínica Alemana, Santiago, Av Vitacura 5951,Vitacura Santiago, Chile, Tel +56997992889, Email naraniss@udd.cl,nadin.aranis@gmail.com

Received: February 23, 2019 | Published: March 01, 2019 human resources trained, reduce the coverage gap of physiotherapy personnel and make efficient use of resources. ${ }^{7}$ This study aims to determine the relationship between the organizational characteristics and the adherence of international recommendations of physiotherapy, in public and private adult ICUs at the national level.

\section{Methods}

Secondary analysis of the database of a cross-sectional study, conducted between 2016-17. ${ }^{8}$ The information comes from $86 \%$ of the adult ICUs that functioned independently from the High Dependency Unit (HDU), and with at least one physiotherapist who worked within the unit, available to answer a 25 -question survey by telephone.

Variables of interest were grouped into two dimensions: (1) organizational characteristics: origin of the ICU (public or private centers), number of beds, level of complexity (low, medium or high), technical-administrative dependence (belong to the Critical Patients Unit, the physical therapy service or external company), and (2) adherence to international recommendations: 24 hours a day and 7 days a week physiotherapist (24/7-PT) availability, physiotherapist/ patient ratio of 1 every 5 ICU beds and presence of physiotherapy specialists. 
An inferential analysis was performed with the statistical test Chisquare and Mann-Whitney, and an exploratory analysis was carried out using multivariate logistic regression to identify factors associated with meeting the international recommendations. This study was approved by the Scientific Ethics Committee of the Medical Staff at the Clínica Alemana Universidad del Desarrollo in Santiago, Chile (approval certificate 2018-29).

\section{Results}

Out of 64 adult ICUs, $59 \%(n=38)$ correspond to public and $41 \%$ $(\mathrm{n}=26)$ to private centers. $70 \%(\mathrm{n}=45)$ healthcare centers with $24 / 7$ PT availability were identified ( $73 \%$ public and $27 \%$ private). The physiotherapist/patient ratio recommended was fulfilled in greater proportion in day shifts during weekdays in the private sector $(50 \%$, $n=13)$ and in the $41 \%(n=26)$ of all Chilean ICUs. The presence of physiotherapy specialists was detected in $31 \%(\mathrm{n}=8)$ of the private sector, versus $18 \%(n=7)$ of the public one.

There were significant differences between the median of the number of beds and the adherence to 24/7-PT availability (12 vs 6 beds in centers with and without 24/7-PT availability, $\mathrm{p}<0.001)$ and also between the median of the number of beds and the maximum of 5 patients per physiotherapist ( 7.5 vs 11 beds in ICUs that have and do not have a physiotherapist/patient ratio of 1 every $5 \mathrm{ICU}$ beds, $\mathrm{p}=0.034$ ). The probability that a unit complies with $24 / 7-\mathrm{PT}$ availability differs significantly depending on the physiotherapists who belong to the Critical Patients Unit vs the physical therapy service or external company $(\mathrm{p}<0.001)$. No significant relationship was found between the level of complexity of the ICU and the adherence to the three international recommendations.

The probability of a Chilean ICU to have 24/7-PT availability is significantly greater $(\mathrm{OR}=5.21,95 \% \mathrm{CI} 1.37-19.81, \mathrm{p}=0.015)$ if it has a public administration and it is 6.59 times higher $(95 \% \mathrm{CI} 1.41$ $30.80, p=0.016)$ if physiotherapists belonged to the Critical Patients Unit instead of to other service models. It is also observed that the probability of having 24/7-PT availability increases in 1.13 times per ICU bed (95\%CI 0.99-1.29), which is not significantly ( $\mathrm{p}=0.060)$.

\section{Discussion}

Chilean ICUs showed greatest adherence to 24/7-PT availability, whereas the physiotherapist/patient ratio and the specialty level presented a compliance that does not exceed $50 \%$ at all. The number of beds and technical-administrative dependence of the physiotherapist unit seem to be relevant in the probability that an ICU complies 24/7PT availability.

Based on internationally available studies, Chile, has greater physiotherapist availability in ICU than what has been reported in other countries; India (24\% physiotherapist availability throughout the night and $79 \%$ on-call), ${ }^{9}$ the United States (1\% of hospitals affiliated with universities and $3 \%$ of community hospitals provided physiotherapist availability at night until 2009) ${ }^{10}$ and Australia (90\% physiotherapist availability during the week and $66 \%$ on weekends). ${ }^{11}$ Literature that reports physiotherapist/patient ratio is scarce. In Greece, a study evidenced that less than half $(9 / 19)$ of their ICUs met the ESICM recommendation, ${ }^{12}$ and found it is directly related to the accomplishment of the optimal range of 6 to 8 beds per ICU recommended by Valentín et al. ${ }^{5}$ Therefore, the number of physiotherapy sessions performed by patient in the ICU might contribute to the adherence to 24/7-PT availability, since it gets necessary to hire human resource to meet the demand for care on an ongoing basis. Nationwide, the adherence of ICUs to specialized trained physiotherapist recommendation was low. Developing a method to assess the competence of clinicians is essential to contribute to the current challenge of translating knowledge into clinical practice through specific professional training, long-life education and competency evaluation. ${ }^{13}$

Campbell et al. compared three management structures used to provide physiotherapy service in the ICU; showing-significant differences only in relation to the number of ICU beds according to the management structure; with a greater number of beds in the model organized according to service areas. ${ }^{14}$ The level of complexity of the unit, which did not show significant difference for the compliance of the recommendations, may be the result of having mainly highly complex ICUs (level III) in Chile.

To reduce reporting bias due to the extraction of information through a telephone survey, a quality control of the database was carried out, biological plausibility of the results was determined and the questions asked were objective limiting the answer to the real practice. A limitation of this study is that the results obtained do not allow attribution of a direct causality. Conversely, a response rate of $86 \%$ constitutes a strength.

\section{Conclusion}

This study demonstrates low adherence to the international recommendations of physiotherapy in ICU nationwide, except for 24/7-PT availability. Thus, it is recognized that the origin of the ICU and the technical-administrative dependence seem to be relevant for its compliance. Future studies should be aimed at identifying the causes of this relationship, in order to favor the management for the hiring of human resources in the ICU throughout the country.

\section{Acknowledgments}

None

\section{Conflicts of interest}

The authors declare no conflicts of interest.

\section{References}

1. Adler J, Malone D. Early mobilization in the intensive care unit: a systematic review. Cardiopulm Phys Ther J. 2012;23(1):5-13.

2. Stiller K. Physiotherapy in intensive care: An updated systematic review. Chest. 2013;144(3):825-847.

3. Castro AAC, Serón P, Fan E, et al. Effect of early rehabilitation during intensive care unit stay on functional status: Systematic review and meta-analysis. PLoS One. 2015;10(7):1-21.

4. Kayambu G, Boots R, Paratz J. Physical therapy for the critically ill in the ICU: a systematic review and meta-analysis. Crit Care Med. 2013;41(6):1543-1554.

5. Valentin A, Ferdinande P. Recommendations on basic requirements for intensive care units: Structural and organizational aspects. Intensive Care Med. 2011;37(10):1575-1587.

6. Saez E. Guías 2004 de organización y funcionamiento de unidades de pacientes críticos. Rev Chil Med Intensiva. 2004;19(4):209-223. 
7. Castillo L. Nivel de complejidad en atencion cerrada; 2012.

8. Merino OC, Leppe J, Molina J, et al. Kinesiólogo 24/7 en Unidades de Cuidados Intensivos en Chile: cobertura y costos asociados. 2017;(October)

9. Kumar J, Maiya A, Pereira D. Role of physiotherapists in intensive care units of India: A multicenter study. Indian $J$ Crit Care Med. 2007;11(4):198-203.

10. Hodgin KE, Nordon CA, Mcfann KK, et al. Physical Therapy utilization in intensive care units: results from a national survey. Crit Care Med. 2009;37(2):561-568.
11. Chaboyer W, Gass E, Foster M. Patterns of chest physiotherapy in australian intensive care units. J Crit Care. 2004;19(3):145-151.

12. Grammatopoulou E, Charmpas TN, Strati EG, et al. The scope of physiotherapy services provided in public ICUs in Greece: A pilot study. Physiother Theory Pract. 2017;33(2):138-146.

13. Pawlik AJ, Kress JP. Issues affecting the delivery of physical therapy services for individuals with critical illness. Phys Ther. 2013;93(2):256265.

14. Campbell L, Bunston R, Colangelo S, et al. The provision of weekend physiotherapy services in tertiary-care hospitals in Canada. Physiother Canada. 2010;62(4):347-354. 